\title{
Nuestra variedad lingüística como variedad biológica ${ }^{1}$
}

Danilo Salamanca

Bluefields Indian and Caribbean University (bicu)

\begin{abstract}
resumen
El estudio se centra en señalar problemas esenciales sobre las consecuencias culturales ante la pérdida de idiomas ancestrales, en particular referido al patrimonio lingüístico indígena centroamericano. Señala algunas relaciones entre este proceso y las discusiones sobre la protección de los ecosistemas biológicos de la región, y llama la atención sobre las políticas que existen o deberían existir, para recuperar y preservar tanto el componente cultural (los idiomas) como el natural.
\end{abstract}

\begin{abstract}
This study examines essential problems related to the cultural consequences due to the loss of ancestral languages, especially Central American Indigenous languages. It mentions relationships between this process and discussions about the protection of biological ecosystems in the region, and addresses policies that exist, or should exist, to recover and preserve both cultural (linguistic) and natural components.
\end{abstract}

Palabras clave: idiomas indígenas, lingüística mesoamericana, políticas lingüísticas, biiodiversidad

Keywords: Indigenous languages, Meso-American linguistics, linguistic policies, biodiversity

1 Versión revisada de la ponencia leída en el I Simposio Internacional del Programa de Lingüística Centroamericana (PROLINCA) Centroamérica: un microcosmo lingüístico, llevado a cabo los 7 y 8 de mayo 2012, en la ciudad de Heredia, en el campus Omar Dengo de la Universidad Nacional de Costa Rica. Recibido: 6 de febrero de 2012; aceptado: 6 de marzo de 2012.

2 Centro de Investigaciones y Documentación de la Costa Atlántica (cidca). Correo electrónico: danilo.salamanca@yahoo.com 


\section{Preámbulo}

En la primera sección, para contextualizar la discusión contenida en las tres secciones siguientes, se efectúa un inventario de la región centroamericana en el momento del contacto con los europeos, así como de la distribución geográfica de los que todavía sobreviven y el número aproximado de los hablantes que quedan. En la segunda sección, de manera general se trata los efectos de la pérdida de los idiomas ancestrales. La tercera sección contiene el tema por evaluar con detenimiento: el potencial valor que nuestros idiomas ancestrales, en tanto creaciones naturales y espontáneas, tienen para la comprensión de las facultades mentales de nuestra especie. En la cuarta sección, la importancia y significado de la pérdida de los idiomas ancestrales examinados en las secciones anteriores pone en contexto de la región centroamericana lo que lleva a recomendar el impulso y desarrollo del programa de trabajo de la recientemente creada Asociación de Lingüística Centroamericana (acaling).

\section{Los idiomas indígenas hablados en el istmo: localización al mo- mento del contacto con los conquistadores españoles y en el pre- sente; el número de sus hablantes ${ }^{3}$}

Pongamos, antes todo, en la mesa ciertos datos significativos acerca de los idiomas indígenas que tenemos, que nos puedan servir de marco para el análisis en este trabajo. Si acudimos a los datos extraídos y a su comparación de los mapas elaborados por Hall y Pérez Brignoli, «Indian Ethnic Groups on the Arrival of the Spanish

3 Fuentes generales utilizadas para esta sección: Lyle Campbell, American Indian Languages. The Historical Linguistics of Native America (Oxford: Oxford University Press, 1997). Lyle Campbell y William Poser, Language Classification: History and Methods (Cambridge, RU: Cambridge University Press, 2008). Adolfo Constenla Umaña, Las lenguas de la Gran Nicoya, Vínculos (Revista de Antropología del Museo Nacional de Costa Rica) 18-19 (1994): 191-208. Adolfo Constenla Umaña, Las lenguas del área intermedia: introducción al estudio areal (San José: Editorial de la Universidad de Costa Rica, 1991). Juan Diego Quesada, The Chibchan Languages (Cartago: Editorial Tecnológica de Costa Rica, 2007). 
Conquest» $\mathrm{y}$ «Indians, 1980s» ${ }^{4}$, podemos llegar a algunas conclusiones interesantes. Por ejemplo, se muestra que buena parte de los idiomas indígenas que se hablaban al momento de la llegada de los españoles ha desaparecido. Los idiomas más afectados fueron los hasta entonces más importantes, hablados por la mayoría de la población: el huetar en Costa Rica; el chorotega en Nicaragua; el lenca en Honduras y en el Salvador. La sociedad colonial fue una sociedad esclavista en la que los indígenas eran una mercancía, y los más accesibles y explotados fueron, generalmente, los grupos lingüísticos más numerosos y mejor organizados, los grupos que eran hegemónicos en cada una de las provincias en que fue dividido el territorio de Centroamérica 5 .

Eran grupos hegemónicos que tenían que ser sometidos y aculturados en primer lugar. Una excepción son los idiomas mayas de Guatemala, que han sobrevivido hasta nuestros días. También hay en El Salvador unos cuantos hablantes de la variedad pipil o nawat del idioma de los aztecas, el náhuatl, del que también se habló otro dialecto - llamémoslo nicarao- ya desaparecido, en Nicaragua ${ }^{6}$. Los lencas y los matagalpas, ambos de origen macrochibcha o chibchense, que se menciona en el mapa reciente porque han conservado en gran parte sus costumbres y su identidad indígena, perdieron sus lenguas desde hace ya al menos un siglo: para Walter Lehmann ${ }^{7}$, que visitó la región durante la primera década del siglo xx con ese objetivo, resultó muy difícil encontrar remanentes de estos idiomas. Según los mapas, los idiomas y culturas más afectadas son las que

4 Carolyn Hall y Hector Perez Brignoli, con el cartógrafo John V. Cotter, Historical Atlas of Central America (Norman: University of Oklahoma Press, 2003).

5 Robert Carmack, Gloria Lara Pinto y Fernando Cruz Sandoval, Los indios de Centroamérica (Madrid: MAPFRE, 1996).

6 W. R. Fowler, Jr., The Pipil Nicarao of Central America (Norman: University of Oklahoma Press, 1989). Jorge Lemus, «Un modelo de revitalización lingüística: el caso del náhuat/pipil del Salvador», Wani: revista del Caribe nicaraguense 62 (2010).

7 Walter Lehmann. Zentral-Amerika, Teil I, Die Sprache Zentral-Amerika in ihren Beziehungen zueinander sowie zu Sud-Amerika und Mexiko (2 vol.) (Berlín: Verlag Dietrich Reimer, 1920). Walter Lehmann, «Ergebnisse einer Forschungreise in Mittelamerika und México 1907-1909», Zeitschrift fur Ethnologie (Jahrg. 421910. Heft 5): 687-749. 
se encontraban del lado Pacífico y mesoamericano del istmo, la zona donde los españoles se implantaron más sistemáticamente, para explotar la mano de obra indígena más numerosa y las tierras más fértiles: en Nicaragua, los chorotegas, subtiavas y nawat; en Honduras los lencas; y en el Salvador lencas y pipiles.

Como consecuencia de eso, los idiomas que sobreviven todavía - con la excepción de los idiomas mayas y los últimos hablantes del pipil salvadoreño - son en su gran mayoría idiomas de estirpe chibchense cuyos grupos, mucho menos numerosos, ocupaban la vertiente atlántica de la región y todo el territorio del actual Panamá. La presencia de los garífunas en Centroamérica es el resultado de un desplazamiento forzado, a partir de la isla antillana de Saint Vincent durante el período colonial ${ }^{8}$.

\section{Nuestro patrimonio lingüístico}

Para complementar la información reflejada en los mapas proporcionamos a continuación una lista de los idiomas por país con el número de personas que los hablan.

Guatemala: idiomas mayas (más de 5 millones de hablantes), garífuna (un pequeño número de hablantes)

Belice: $\quad$ inglés criollo $(70.000)^{9}$, garífuna (16.000)

El Salvador: pipil (varias decenas de hablantes)

Honduras: garífuna (40.000), miskito (50.000), inglés criollo (12.300), tawahka: sumus (500), pech (600), tolupán o tol (300)

Nicaragua: miskito (120.000), twahka-panamahka (10.000), ulwa (500) rama (30)

8 Andrade de Coehlo y Ruy Galvao, Los negros caribes de Honduras (2a ed. en español, 1a ed. de 1955) (Tegucigalpa: Guyamuras, 1995). Genevieve Escure y Armin Schwegler, «Garifuna in Belize and Honduras», Creoles, Contact, and Language Change: Linguistic and Social Implications (Amsterdam/Philadelphia: John Benjamin, 2004).

9 John Holm (Ed.), Central American English (Julios Groos Verlag: Heidelberg, 1983). 
Costa Rica: guatuso (350), bribris (6.000), cabécar (2.500), boruca (extinto)

Panamá: $\quad$ guaymí, moveré o ngobe (150.000), buglere-bokota (2.500), teribe (1.500), kuna (60.000), emberá(6.000), waunaan (2.000)

Debido a la dificultad para obtener datos fidedignos sobre las lenguas y sus usuarios, los números son aproximados y tienen como principal fin ofrecer una idea de los porcentajes del total de la población en las naciones respectivas, y de los tamaños relativos de las comunidades de hablantes para cada una de las lenguas indígenas sobrevivientes.

\section{¿Qué se pierde cuando se pierden los idiomas autóctonos de la región?}

Este tema puede ser abordado según varias aproximaciones. La homogenización lingüística y cultural fue considerada durante mucho tiempo un progreso según las autoridades que gobernaron el imperio español; todavía en nuestros tiempos, hay quienes piensan -incluso entre los mismos hablantes, por ejemplo los padres de familia en relación a sus hijos - que la conservación de la lengua y cultura ancestral puede entorpecer el desarrollo de la nación y ser desventajosa en la vida personal de un individuo.

Si esto es cierto o no depende de las circunstancias políticas y sociales que se establezcan para ellos. Actualmente, los miskitos tienen en Nicaragua un lugar privilegiado en la Región Autónoma del Atlántico Norte (lo mismo es cierto para los otros indígenas habitantes de las regiones autónomas); prueba de ello es que todos los habitantes de la región, incluso los que antes los despreciaban y que no hablan su lengua, ahora quieren ser considerados —al menos legalmente- miskitos ${ }^{10}$.

10 Danilo Salamanca, «Los dos rostros indígenas de Nicaragua y Centroamérica», Wani: revista del Caribe nicaragüense 65 (2012). 
Independientemente de las desventajas prácticas que el estatus de indígena y el pobre conocimiento del español puede representar, hay razones generalmente aceptadas, esgrimidas a favor de la conservación de los idiomas indígenas ancestrales. Sin pretender ser exhaustivos y sin profundizar en los detalles, vamos a hacer una lista de algunas de las principales en esta sección, para después concentrarnos en la siguiente sección sobre el tema del presente estudio, el interés de conservar la evidencia científica que estos idiomas constituyen para investigar el funcionamiento de nuestra capacidades biológicas relativas al lenguaje.

El idioma como elemento legitimante de los derechos de los indígenas, en particular el derecho a la tierra donde viven

En las luchas de las comunidades indígenas que viven en los territorios ancestrales de sus antepasados - lucha feroz que no pocas veces conduce a actos de violencia y asesinatos - la conservación de la lengua es un elemento que tiene un lugar importante. En muchas partes del istmo, la mezcla racial ha hecho que el aspecto físico o la herencia biológica hayan dejado de ser un factor de identificación de la población indígena en relación a los mestizos y otros habitantes de las naciones respectivas.

Una de las razones presentadas para deslegitimar los reclamos de derechos territoriales y otros de las comunidades indígenas es que estas no son más descendientes de los habitantes ancestrales que el resto de la población. En ese contexto, la conservación de los idiomas desempeña un papel parecido a la conservación de un título de propiedad. Los ejemplos sobran en todas partes del istmo, y las consecuencias de haber perdido los idiomas ancestrales están a la vista.

El idioma como reservorio de conocimientos sobre el medio ambiente y las culturas ancestrales

Los idiomas reflejan en la manera de clasificar las plantas y animales y en el vocabulario referente a la naturaleza, los conocimientos 
acumulados sobre el medio ambiente y los fenómenos naturales propios de las regiones ancestrales de los indígenas. Los idiomas son también el vehículo por el cual se transmite la cultura, el arte y la visión del mundo que fueron desarrolladas a lo largo de su historia por los pueblos ancestrales. Son para nosotros, sus descendientes y actuales habitantes de sus territorios, una preciosa parte de nuestro patrimonio cultural. Ambas, cultura y ciencia, tienden a perderse cuando el idioma se muere.

\section{El atractivo turístico que representan las culturas ancestrales}

Las lenguas son el reservorio de los conocimientos, las costumbres y creencias de nuestras culturas ancestrales. Para conservar mejor las culturas conviene incentivar la conservación de las lenguas. El turismo lingüístico, propiamente, es todavía incipiente en Centroamérica, aunque hay escuelas de idiomas mayas en Antigua, Guatemala, desde hace más de veinticinco años. Pero la atracción de las culturas indígenas y sus creaciones artesanales y arquitectónicas (las ciudades mayas, en particular) son uno de los principales atractivos turísticos de la región.

Aparte de Guatemala, el país más rico al respecto, países como Nicaragua, Honduras o Panamá, donde grandes segmentos de los territorios nacionales son habitados por poblaciones indígenas, poseen como atracción turística las culturas sobrevivientes de nuestros antepasados.

No es aquí el lugar de evaluar las consecuencias, o para profundizar sobre las formas y maneras en que el desarrollo del turismo cultural puede afectar a los indígenas y las economías de nuestros empobrecidos países; baste decir que es todavía una razón adicional para querer conservar los idiomas.

Tales son algunas de las razones que demandan un esfuerzo deliberado y efectivo para impedir el deterioro y desaparición de nuestros idiomas ancestrales. Existen otras, y en la sección siguiente abordaremos una que - aunque tal vez menos importante - merece ser analizada con atención. 


\section{La variedad lingüística como variedad biológica ${ }^{11}$}

Una relación íntima y potencialmente reveladora pareciera existir entre la protección de los idiomas en peligro y la protección de la biodiversidad.

\section{Las culturas ancestrales como protectoras del ambiente}

Existe una tendencia a que en los territorios donde los idiomas ancestrales han sido conservados, la biodiversidad es mayor. Es frecuente que las culturas y lenguas en peligro sobreviven en reservas biológicas de diverso tipo. En cierto sentido, la sobrevivencia de los grupos étnicos originales es parte de la biodiversidad conservada en los territorios donde se localizan.

La presencia de grupos humanos que han conservado sus culturas y sus lenguas ancestrales no solo es, por así decirlo, testigo como lo son las especies en peligro- de que ciertos territorios se han

conservado relativamente protegidos de las influencias externas vigentes fuera de ellos, sino también actúan como garantes o protectores de esos territorios. Las especies en peligro no pueden protestar, pero los indígenas sí, sobre todo cuando hay un marco legal que lo permita.

Por ello, en el transcurso de 2012, hemos visto protestar a los hablantes de ngobe en Panamá ante las concesiones mineras e hidroeléctricas en su territorio; a los hablantes de malecu o guatuso en la zona fronteriza entre Nicaragua y Costa Rica por la apertura de una trocha o carretera a lo largo de la margen costarricense del río San Juan, y a los mayagnas en la reserva forestal de Bosawas en el norte de Nicaragua por la extracción ilegal de madera. En los lugares donde sobreviven, los hablantes de idiomas indígenas denuncian los daños al ambiente y luchan por la defensa de sus territorios, que es

11 Juan C. Martínez Sánchez, Jean Michel Maes, Eric van de Berghe, Slavadora Morales y Edgar A. Castañeda, Biodiversidad zoológica en Nicaragua (Managua: Marena/PNUD/Helios, 2001). Ministerio del Ambiente y Recursos Naturales, Biodiversidad en Nicaragua: un estudio de país (Managua: MARENA-PANIF/CIRA, 1999). 
también la defensa de su propia supervivencia y modo de vida. La diversidad biológica y la diversidad cultural y lingüística están, de esa manera, ligadas.

El mencionado interés turístico con relación a la conservación de las culturas y las lenguas indígenas, está ligado a la conservación de su hábitat natural, las reservas de biodiversidad, que constituyen también un atractivo turístico por sí solas.

Esta es ya una primera razón para conservar las culturas y las lenguas ancestrales, que son garantes y protectoras de la diversidad biológica. Pero ¿cuál es el auténtico interés de conservar la diversidad biológica en el planeta?

\section{Los argumentos a favor de la conservación de la diversidad biológica $^{12}$}

Podría argumentarse a favor de la conservación de la diversidad biológica. Hay una especie de instinto moral (biofilia) que inclina a querer impedir la aniquilación de los seres vivos; es cierto que hay razones objetivas y egoístas, como veremos adelante, que deberían inclinarnos en esa dirección. Es ese instinto que aducen los activistas que desarrollan campañas para proteger especies animales que en algunos casos nunca hemos visto ni vamos a ver.

Es difícil determinar experimentalmente el número e identidad óptimos de especies en un ambiente determinado para maximizar su equilibrio y productividad de efectos benéficos, comoquiera que estos últimos sean definidos. La cuestión es debatible, y debatida, en la literatura especializada. En ese contexto, una conclusión prudente es que lo mejor sería conservar todas las especies que se hallaban y se hallan en los sectores del planeta donde ha habido menos intervención humana; es decir, conservar al máximo la biodiversidad que encontramos. El razonamiento es que el equilibrio biológico que encontramos es el resultado de un proceso evolutivo de intentos y

12 Esta sección está ampliamente basada en Kevin Gaston J. y John I. Spicer, Biodiversidad: Introduction (Oxford: Blackwell, 2004). 
errores por parte de la naturaleza para maximizar la vida en un espacio determinado.

Pero además, se pueden encontrar valores utilitarios y no utilitarios para conservar un máximo de especies. Los repasaremos a modo de contexto para la discusión siguiente sobre las lenguas en peligro y la variedad lingüística.

Dentro de los valores utilitarios directos que las especies animales y vegetales nos aportan -se trata de beneficios cuantificables que pueden incluso ser evaluados monetariamente- se cuentan los siguientes: alimentos, medicinas, control biológico (ciertas especies, como los gatos, pueden servirnos para contrarrestar los efectos negativos de otras, como los ratones, evitando el uso de pesticidas), materiales industriales, mascotas y plantas ornamentales, ecoturismo ${ }^{13}$. Como valores utilitarios indirectos (también cuantificables monetariamente), los ecosistemas naturales y equilibrados y las diferentes especies animales y vegetales tienen un efecto sobre la regulación atmosférica, la regulación climática, la regulación hidrológica, el ciclado de nutrientes, la polinización, la fotosíntesis y la formación y mantenimiento de los suelos, entre otros. Se han señalado aportes o valores no utilitarios, no cuantificables, también razones importantes para querer conservar la biodiversidad. El primero de ellos es el valor intrínseco. Muchas sociedades estiman que, independientemente del uso o beneficio que los humanos puedan sacar de los otros seres vivos, hay un principio moral o religioso, tal vez biológicamente condicionado en nuestra especie, que nos obliga a respetar la tierra, la naturaleza y las diferentes formas de vida. El valor de legado, a su vez, se puede formular de la siguiente manera: debemos compensar

13 Christien Duverger, Mesoamérica. Arte y antropología, trad. Aurelia Álvarez (México: Consejo Nacional para la Cultura y las Artes-Landucci, 2000). George Hasemann, La etnología y la lingüística en Honduras, una mirada retrospectiva (Estudios Antropológicos e Históricos, 9) (Tegucigalpa: Instituto Hondureño de Antropología e Historia, 1991). George Hasemann y Gloria Lara Pinto, La zona central: regionalismo e interacción, Robert Carmack (Ed.), Historia general de Centroamerica. Tomo 1. Historia Antigua (Madrid: Ciruela, 1993): 135-216. 
a nuestros hijos en el futuro por la pérdida de riquezas biológicas de las cuales somos responsables.

Finalmente, y lo hemos dejado de último porque quisiéramos retomarlo en la sección siguiente, la conservación de las formas de vida existentes representan un valor de opción, porque no sabemos cuáles pueden ser sus posibles usos benéficos en el futuro. Bajo este rubro cabe la posibilidad de que ciertas especies o formas de vida puedan servirnos en el futuro para un uso científico, porque nos pueden proporcionar información que necesitamos para entender el mundo y por eso nos conviene conservarlas.

\section{Los idiomas ancestrales como seres vivos: el valor de opción de la variedad lingüística}

Quisiéramos abordar en esta sección a los idiomas aborígenes como entidades vivas, que pueden morir, y que son parte de la biodiversidad existente en el mundo. Desde la perspectiva de la investigación de la variedad biológica, los idiomas naturales son comparables con la variedad de especies de animales y vegetales que encontramos en el planeta y que en su conjunto constituyen la evidencia de la que disponemos para entender la vida y sus manifestaciones.

El lenguaje ha sido identificado desde la antigüedad por filósofos y pensadores como prueba tangible de la paradójica dualidad cuerpo-mente en el ser humano. Por consiguiente, el estudio de las propiedades y el uso, comunes o particulares a cada idioma, puede ser considerado una vía real para acceder al funcionamiento de nuestras facultades mentales, un área para nosotros particularmente interesante del funcionamiento de la vida en nuestro planeta.

Todas las teorías lingüísticas existentes se interesan en el sustrato biológico del lenguaje. En la discusión que sigue vamos a utilizar la terminología y los conceptos desarrollados a partir de las investigaciones de Noam Chomsky y su escuela conocida en sus comienzos, y todavía muy ampliamente, como gramática generativa. 
La mayoría de términos y conceptos que serán utilizados pueden ser expresados o al menos son comprensibles en los términos de las principales teorías rivales. El programa de investigación de la gramática generativa persigue, mediante el estudio de las diferentes, las propiedades y condiciones que las lenguas humanas comparten y que por consiguiente podemos suponer están determinadas por el sustrato biológico del lenguaje, la facultad u órgano del lenguaje.

Todos los idiomas naturales existentes afrontan la misma situación: la transmisión de ideas, pensamientos o significados, bajo ciertas condiciones. Los significados son codificados por operaciones mentales en sonidos que son emitidos por los órganos de la fonación, transmitidos por ondas sonoras que son captados por los órganos auditivos y descifrados por operaciones mentales que recuperan el significado. A pesar de las diferencias que podrían parecer espectaculares, la teoría lingüística supone y ha logrado confirmar en buena medida que, a pesar de las apariencias, todos los idiomas realizan esencialmente las mismas operaciones y están sometidos a las mismas condiciones. Sin embargo, hay diferencias que constituyen la evidencia por la cual podemos aproximarnos a una formulación más abstracta y más exacta de lo que realmente está en juego en el lenguaje; es decir, el funcionamiento de la facultad mental y de los instrumentos periféricos de producción y recepción que lo permiten y condicionan.

Desde ese punto de vista, cada uno de los idiomas existentes en el mundo es susceptible de aportar precisiones que nos permitan refinar la teoría. Esto es particularmente cierto de las familias de idiomas que han sido menos estudiadas. Al igual que ocurre con las especies animales y vegetales, entre dos especies de ratones la variedad o variación es menor que entre un ratón y un alacrán. La información proporcionada por el estudio de los dos ratones para comprender las maneras en que los seres vivos resuelven problemas similares (alimentarse, moverse...) es menor que la que podemos obtener si estudiamos un ratón y un alacrán. De la misma forma, la información original o diferente que dos lenguas humanas dadas 
pueden aportar es mayor si se trata de lenguas pertenecientes a familias distintas de lenguas que han evolucionado independientemente durante mucho tiempo.

La mayor parte de los estudios en el marco de la teoría de la gramática generativa han sido hechos a partir de un número limitado de idiomas: los grandes idiomas del mundo, y en particular las lenguas europeas. Su análisis ha sido desarrollado en considerable detalle y las propuestas que se han hecho para extraer generalizaciones sobre la facultad del lenguaje a partir de los datos considerados han sido revisadas y verificadas por hablantes nativos que son al mismo tiempo lingüistas profesionales.

Los idiomas aborígenes han quedado generalmente al margen de esas investigaciones de detalle. Esto no es necesariamente un problema, porque la teoría o exploración de la facultad del lenguaje puede avanzar a partir de la consideración de un número limitado de idiomas. A fin de cuentas, lo que sabemos sobre la vida lo hemos aprendido a partir del estudio de un muy pequeño porcentaje de las especies existentes.

La mejor comprensión del funcionamiento de la facultad del lenguaje sería beneficiosa para la humanidad. Las utilizaciones potenciales van desde el tratamiento de los diferentes disfuncionamientos del lenguaje que se presentan naturalmente o por lesiones en el cerebro, hasta el perfeccionamiento de medios de comunicación artificial.

En Centroamérica se encuentran vivos todavía varios miembros de familias de idiomas que solo existen en la región y en sus fronteras (chibcha, misumalpa, maya y chocó, además de otros idiomas no clasificados o muy tenuemente presentes) y que tienen propiedades muy particulares. Por consiguiente, existe el potencial de hacer aportes al estudio de la mente y el lenguaje a partir de estos idiomas; potencialmente son un tesoro de conocimientos.

Esta es, entonces, una razón suplementaria para querer preservar estos idiomas: son parte del patrimonio biológico de la región. 


\section{Conclusión: la necesidad de un programa centroamericano de investigaciones lingüísticas y la protección de nuestro patrimo- nio lingüístico}

\section{El problema}

Hemos repasado varias razones por las cuales la preservación de nuestro patrimonio lingüístico centroamericano es importante. Una serie de razones generales fueron presentadas brevemente (en la segunda sección) y un intento de iluminar mutuamente las pérdidas respectivas que ocasiona la disminución de la diversidad biológica, en general, y la pérdida de la diversidad lingüística, en particular, fue realizado en la tercera sección.

Si lo que hemos defendido y argumentado anteriormente es cierto, ya que estamos conscientes de la gravedad e importancia del problema estamos obligados a examinar qué medidas se están tomando y que medidas podemos tomar para luchar contra la degradación y pérdida de nuestros idiomas.

\section{Lo que se hace}

En cada uno de nuestros países, dependiendo en gran parte de las luchas de los indígenas nacional e internacionalmente, los gobiernos se han visto forzados a tomar medidas más o menos efectivas en la dirección de preservar las lenguas. Pero las medidas decididas casi nunca han sido implementadas adecuadamente, y cuando lo son, es improbable que sean suficientes; necesitamos hacer más, y parte de lo que tenemos que hacer se ubica en un ámbito internacional.

\section{Lo que podemos y debemos hacer}

Debido a la implantación de las lenguas individuales en varios países (como el ngobe, el miskito, el sumo, el chortí, el náhuatl, el garífuna, el inglés criollo, e incluso el mismo español); y más aún, la implantación de las familias de lenguas como los idiomas chibchas 
y chibchenses o los idiomas oto-mangues, es conveniente considerar la región como un solo espacio de estudio.

La escasez de recursos materiales y humanos en cada país es otra razón que obliga a la unión de los lingüistas centroamericanos para afrontar la tarea de preservar nuestro patrimonio lingüístico.

Por razones prácticas, las limitaciones en recursos materiales y humanos y la implantación de las lenguas y familias de lenguas en varios países, pero también por razones científicas, para poder abordar temas transversales a los idiomas y para poder reunir un máximo de evidencia pertinente a la solución de problemas e interrogantes, es conveniente e incluso necesario desarrollar un programa de estudios lingüísticos centroamericanos ${ }^{14}$.

14 La Asociación Centoamericana de Lingüística (acaling) fue creada para ese fin. 